\title{
Capsule Commentary on McCoy Et al. Hospital Readmissions Among Commercially-Insured and Medicare Advantage Beneficiaries with Diabetes and the Impact of Severe Hypoglycemic and Hyperglycemic Events
}

\author{
Andrew R. Zullo, PharmD ScM PhD \\ Department of Health Services, Policy, and Practice, Brown University School of Public Health, Providence, RI, USA.
}

$\mathrm{J}$ Gen Intern Med 32(10): 1132

DOI: $10.1007 / \mathrm{s} 11606-017-4109-8$

(c) Society of General Internal Medicine 2017

$\mathrm{T}$ his retrospective cohort study by McCoy et al. ${ }^{1}$ used administrative data from commercially insured and Medicare Advantage beneficiaries with diabetes to describe the relative frequency of hospital readmissions for severe dysglycemia (hypoglycemia and hyperglycemia) and other causes. It also examined potential predictors of such events. The authors found that the all-cause 30-day readmission rate was $10.8 \%$. Heart failure was the most common cause for readmission $(8.9 \%)$, and severe dysglycemia accounted for $2.5 \%$ of readmissions ( $38.3 \%$ hyperglycemia, $61.0 \%$ hypoglycemia, $0.7 \%$ unspecified). Younger age, history of dysglycemia, and a higher Diabetes Complications Severity Index score were independent predictors of severe dysglycemia relative to other readmission causes.

Most studies of dysglycemia using administrative data ${ }^{2}$ have been limited to the assessment of severe events because mild-to-moderate events are (1) most likely to occur in settings outside of clinical care and (2) are unlikely to be recorded as justification for a clinical encounter or payment of health services. The epidemiology and consequences of mild-tomoderate dysglycemia are therefore unaddressed by this study and are much less well understood in the empirical literature. ${ }^{3}$ Future studies in large populations should strive to assess mild-to-moderate dysglycemia and build on administrative data by leveraging the information in electronic health records, including both structured data fields and free-text clinical notes. ${ }^{3}$ These additional data will help to provide a more complete understanding of dysglycemia that best informs optimal transitional and ambulatory care practices and permits targeting of interventions to individuals with diabetes at highest risk.

As the authors point out, the absence of frailty measures in their study is a major limitation and complicates the

Published online June 26, 2017 interpretation of their conclusion that younger individuals are at highest risk for severe dysglycemia admissions. Even if severe dysglycemia events are more common in younger individuals, the consequences of such events are likely more catastrophic for frail older adults. ${ }^{4}$ This is especially true for individuals residing in long-term care settings, where medication regimen complexity is greater and insulin use is more common. Studies of dysglycemia in frail older adults are acutely needed as data on the epidemiology and consequences of occurrence are lacking. ${ }^{5}$

Corresponding Author: Andrew R. Zullo, PharmD ScM PhD; Department of Health Services, Policy, and PracticeBrown University School of Public Health, Providence, RI, USA (e-mail: andrew_ zullo@brown.edu).

\section{Compliance with Ethical Standards:}

Conflict of Interest: The author has no conflicts of interest with any of the material in this manuscript.

\section{REFERENCES}

1. McCoy RG, Lipska KJ, Herrin J, Moore Jeffery M, Krumholz HM, Shah ND. Hospital readmissions among commercially-insured and medicare advantage beneficiaries with diabetes and the impact of severe hypoglycemic and hyperglycemic events. Journal of General Internal Medicine. 2017; doi:10.1007/s11606-017-4095-x.

2. Edridge CL, Dunkley AJ, Bodicoat DH, Rose TC, Gray LJ, Davies MJ, et al. Prevalence and incidence of hypoglycaemia in 532,542 people with type 2 diabetes on oral therapies and insulin: a systematic review and meta-analysis of population based studies. Plos one. 2015; 10(6):e0126427.

3. Nunes AP, Yang J, Radican L, Engel SS, Kurtyka K, Tunceli K, et al. Assessing occurrence of hypoglycemia and its severity from electronic health records of patients with type 2 diabetes mellitus. Diabetes Research and Clinical Practice. 2016;121:192-203.

4. Munshi MN, Florez H, Huang ES, Kalyani RR, Mupanomunda M, Pandya N, et al. Management of diabetes in long-term care and skilled nursing facilities: a position statement of the American Diabetes Association. Diabetes Care. 2016;39(2):308-18.

5. Zullo AR, Dore DD, Gutman R, Mor V, Smith RJ. National glucoselowering treatment complexity is greater in nursing home residents than community-dwelling adults. Journal of the American Geriatrics Society. 2016;64(11):e233-e5. 